\title{
Analysis of the Severe Maternal Outcomes between Resource-Poor and Resource-Rich Hospitals in China's Hunan Province from 2012 to 2018
}

\author{
Lili Xiong $\mathbb{D}$, Mengjun Zeng $\mathbb{D}$, Aihua Wang $\mathbb{D}$, Donghua Xie $(\mathbb{D}$, Fanjuan Kong $\mathbb{D}$, \\ and Zhiyu Liu
}

Hunan Province Maternal and Children Care Hospital, China

Correspondence should be addressed to Zhiyu Liu; 415172112@qq.com

Received 17 July 2020; Revised 21 September 2020; Accepted 20 October 2020; Published 12 November 2020

Academic Editor: A.Seval Ozgu-Erdinc

Copyright ( $) 2020$ Xiong Lili et al. This is an open access article distributed under the Creative Commons Attribution License, which permits unrestricted use, distribution, and reproduction in any medium, provided the original work is properly cited.

Background. This facility-based study analyzed the epidemiology and incidence of maternal near miss (MNM) and mortality by hospital level as part of Hunan Province's efforts to raise the quality of hospital care for women. Methods. We used data for MNM and mortality cases for 2012-2018 from 17 hospitals (12 resource-poor facilities, five resource-rich facilities) that receive referrals for obstetric complications in Hunan Province. Data were drawn from China's National Maternal Near Miss Surveillance System and collected using the World Health Organization near miss tool. We calculated the ratio of severe maternal outcomes (SMO) (i.e., MNM and maternal death (MD) cases), mortality index (MI), and MNM to mortality ratio (MNM : MD), and epidemiological factors, organ dysfunction, and maternal complications stratified by hospital level. The chisquare tests to examine differences between groups and total ratios and 95\% CI were calculated. Results. There were 518 SMO cases (489 MNM and 29 MD) among 279407 live births (LBs) and 1299 SMO cases (1262 MNM and 37 MD) among 232386 LBs in resource-poor and resource-rich facilities. The total MNM ratio in resource-poor and resource-rich hospitals was 1.75 (95\% CI: 1.60-1.91) and 5.43 (95\% CI: 5.14-5.74) per 1000 LBs, respectively. There were differences in SMO cases between resource-poor and resource-rich hospitals in maternal age, education, parity, antenatal visits, and history of cesarean sections. In MNM cases, coagulation dysfunction was the main organ dysfunction (resource-poor hospitals: 59.10\%; resource-rich hospitals: 79.32\%), and the main maternal complications were obstetric hemorrhage (resource-poor hospitals: 71.98\%) and hepatopathy (resource-rich hospitals: 69.49\%). For MD cases, the main maternal complications were neurologic dysfunction (resource-poor hospitals: $41.38 \%$ ) and coagulation dysfunction (resource-rich hospitals: $42.55 \%$ ). Anemia was the main maternal complication for SMO cases in both resource-poor (69.69\%) and resource-rich (68.59\%) hospitals. Conclusions. MNM and MD are higher in resource-rich hospitals compared with resource-poor hospitals. The obstetric emergency capacity of resource-rich hospitals is higher than that of resource-poor hospitals. Future government policies should consider upgrading the obstetric emergency treatment capacity in resource-poor hospitals or to redistinguish the social functions of different medical institutions.

\section{Background}

The maternal mortality ratio (MMR) represents the number of maternal deaths per 100000 live births (LBs) and is a core indicator of a country's health and social development. Many countries have reported decreasing MMRs in the context of economic development and improved population health. The Global Burden of Disease study showed that over the last decade, the MMR fell by about
$30 \%$ worldwide, from 288 in 2000 to 196 in 2015 [1]. Maternal mortality also declined by $8.9 \%$ per year between 1997 and 2014 in China, which has achieved Millennium Development Goal 5 (i.e., a 75\% reduction of the MMR between 1990 and 2015) [2]. The MMR in China's Hunan Province declined by $45.24 \%$ between 2009 and 2014 [3]. Today, maternal death (MD) has become an extremely low probability event in the developed countries and parts of the developing countries, although severe maternal 
morbidity or maternal near miss (MNM) cases remain major public health challenges for healthcare systems globally [4]. MNM is defined by the World Health Organization (WHO) as "a woman who nearly died but survived a complication that occurred during pregnancy, childbirth or within 42 days of termination of pregnancy" [5-7]. The concept of MNM as developed by the WHO has been used to assess life-threatening conditions during pregnancy, labor, and puerperium to enable interventions to be focused on the chain of events leading to a woman's near miss or death $[8,9]$. Prevention of MNM is now a major focus in countries with a low MMR, as it offers a means to improve the quality of maternal healthcare [10]. Assessment of pregnant women with severe maternal outcomes (SMO), which includes MNM and MD, may contribute to accelerating the reduction of morbidity and mortality [11]. The WHO recommends that countries should closely monitor SMO trends to identify preventable causes as well as system- and provider-related failures [12].

Progress has been made in reducing $\mathrm{MD}$ in Hunan Province [3]. However, there have been no official reports of SMO or MNM cases in Hunan Province. Additionally, regional disparities in maternal and child health persist in areas of China, particularly in hospitals, and no study has explored the incidence of SMO and MNM in different levels of medical institutions. However, evaluation of SMO is an important indicator that can help to improve quality of maternal care [13].

This study is aimed at comparing the incidence and epidemiological characteristics of SMO cases in selected resource-poor and resource-rich hospitals in Hunan Province, China, from January 1, 2012 to December 31, 2018. It is important to understand the incidence and epidemiology of SMO cases in different institutions to provide a basis for the government when developing policies concerning prevention of maternal deaths and improvement of obstetrical diagnosis and treatment across different levels of medical and health institutions.

\section{Methods}

2.1. Aim. This study is aimed at analyzing the differences in incidence and epidemiological characteristics of SMO cases between resource-poor and resource-rich hospitals in Hunan Province, China, from 2012 to 2018.

2.2. Research Design. This study used a retrospective design based on data for Hunan Province collected from China's National Maternal Near Miss Surveillance System (NMNMSS) for 2012-2018. This study dynamically monitored the occurrence of SMO in different resources of medical institutions.

2.3. Setting. Hunan Province is located in central China, has a population of 71.47 million people, and covers an area of $21.18 \mathrm{~km}^{2}$. This study included data for 12 resource-poor hospitals and five resource-rich hospitals. The 17 hospitals have been the maternal near miss surveillance hospitals of Hunan Province in China's National Maternal Near Miss
Surveillance Program. Resource-rich medical institutions in China are considered better than resource-poor institutions in terms of the organization size, rate of use of obstetric beds, available human resources, and first aid and service capacity.

2.4. Participants' Characteristics. The participants evaluated in this study were pregnant and postpartum women admitted to the obstetrics departments or intensive care units of the studied institutions, and those who died in hospital. We excluded pregnant women hospitalized in departments other than obstetrics and those with minor abdominal pain but no other obstetric complications. Women that required only embryo preservation (e.g., after in vitro fertilization procedures) and traditional Chinese medicine treatment were also excluded.

The study population included all women during pregnancy, childbirth, or within 42 days of termination of pregnancy regardless of age. Identification of MNM cases was based on the presence of 25 organ and system dysfunction criteria (cardiovascular, respiratory, renal, coagulation, hepatic, neurological, and uterine) via clinical-, laboratory-, and management-based parameters. We defined MNM cases as "a woman who nearly died but survived a complication that occurred during pregnancy, childbirth, or within 42 days of termination of pregnancy." MD was defined as "the death of a woman while pregnant, or within 42 days of the termination of pregnancy, irrespective of the duration and site of the pregnancy, from any cause related to, or aggravated by, the pregnancy or its management but not from accidental or incidental causes" [14].

2.5. Data Collection. Data were drawn from China's NMNMSS for a 7-year period from January 1, 2012 to December 31, 2018. The NMNMSS was established in October 2010 and covers 17 health facilities in Hunan Province. The system is aimed at enumerating all MD and MNM cases based on the WHO near miss criteria. Data were collected through a review of all research objects from admission to the obstetrics and gynecology units to discharge. The 17 hospitals were selected by health administration departments of Hunan provincial to monitor causes of child and maternal mortality within the province. The NMNMSS sampling methods have been described in detail elsewhere [15]. Doctors responsible for patient care within each facility were trained to collect and record the main information for maternal cases admitted to the obstetrics departments from admission to discharge. The information collected included maternal sociodemographic characteristics, pregnancy termination conditions or complications, rescue measures and procedures, and maternal and perinatal outcomes during pregnancy, delivery, and postpartum. Collected data for women that met the inclusion criteria were entered into the NMNMSS. Quality assurance was ensured by county, municipal-, and provincial-level maternal and child health hospital staff. If surveillance hospital errors exceed a predefined standard (e.g., if obstetric complications were 
underreported by more than 5\%, MD was underreported by more than $1 \%$, or MNM was underreported by more than $5 \%$ ), they reexamined all data in the process of quality checking.

The primary outcome indicators were SMO ratio, MNM ratio, MMR, and mortality index (MI). SMO cases included MNM and MD cases, and referred to a lifethreatening condition (i.e., organ dysfunction or death). The SMO ratio is the number of MNM cases plus MD cases per 1000 LBs. The MNM ratio refers to the number of MNM cases per 1000 LBs. The MMR represents the number of MD cases per 100000 LBs. The MNM mortality ratio (MNM:MD) refers to the ratio of $M N M$ to $M D$ cases. Finally, the MI refers to the number of MD cases divided by the sum of MNM and MD expressed as a percentage $(\mathrm{MI}=\mathrm{MD} /[\mathrm{MNM}+\mathrm{MD}])$. The $\mathrm{SMO}$ and $\mathrm{MNM}$ ratios give an estimate of the amount of care and resources that would be needed in an area or facility. SMO ratio, MNM:MD, and $\mathrm{MI}$ are indicators for obstetric quality; the higher the $\mathrm{MI}$ and $\mathrm{SMO}$ ratios, more women with life-threatening conditions die (low quality of obstetric care). Conversely, the higher the MNM:MD, fewer women with life-threatening conditions die (better quality of obstetric care).

We reviewed relevant NMNMSS data from January 1, 2012 to December 31, 2018. Next, we exported data from the NMNMSS to IBM SPSS version 20.0 for our analyses. In total, data of 1817 SMO cases from 541128 hospitalized pregnant women were used to analyze in this study. Frequency tables and crosstabulations by resource-poor and resource-rich hospitals were produced based on the collected demographic and clinical variables. All statistical tests were considered significant at $P<0.05$. This study was approved by the Ethic Review Committee of Hunan Province Maternal and Children Health Care Hospital. The requirement for obtaining informed consent was waived because of the retrospective design of this study. This study was carried out in accordance with the principles of the Declaration of Helsinki. Hunan Province Maternal and Children Health Care Hospital is a comprehensive maternal and child care institution responsible for women's and children's healthcare in Hunan Province.

\section{Results}

3.1. Incidence of SMO. During the 7-year period, 541128 pregnant women were hospitalized in the 17 studied hospitals. There were $511793 \mathrm{LBs}$, which resulted in 1817 SMO cases. The total SMO ratio and 95\% confidence interval (CI) in resource-poor hospitals was 1.85 (95\% CI: $1.70-$ 2.02) per $1000 \mathrm{LBs}$ and in resource-rich hospitals was 5.59 (95\% CI: 5.29-5.90) per 1000 LBs. There were 489 MNM cases and $29 \mathrm{MD}$ cases in resource-poor hospitals, and $1262 \mathrm{MNM}$ cases and $37 \mathrm{MD}$ cases in resource-rich hospitals. The total MNM ratio was 1.75 (95\% CI: 1.60$1.91)$ and 5.43 (95\% CI: 5.14-5.74) per 1000 LBs in resource-poor and resource-rich hospitals, respectively. The total MMR was 10.38 (95\% CI: 6.95-14.90) and 15.92 (95\% CI: $11.51-21.95$ ) per 100000 LBs in resource- poor and resource-rich hospitals, respectively. In resource-poor hospitals, the total MI was $5.60 \%$ and the MNM ratio was 16.86 . In resource-rich hospitals, the total MI was $2.85 \%$ and the MNM ratio was 34.11 . A summary of deliveries and SMO and MNM indicators in resourcepoor and resource-rich hospitals is presented in Table 1. We observed that the MNM ratio and MNM:MD in resource-poor hospitals declined from 2014 to 2018, whereas the $\mathrm{MNM}$ ratio, $\mathrm{MNM}: \mathrm{MD}$, and $\mathrm{MMR}$ in resource-rich hospitals fluctuated during the 7-year period (Figure 1).

3.2. Demographic Characteristics of Women with SMO. The majority $(31.15 \%)$ of SMO cases in both resource-poor and resource-rich hospitals occurred among women aged 30-34 years. The highest proportion (48.76\%) of cases was recorded as having a high school education. Only $2.20 \%$ of cases were not married at the time of the investigation, and nearly half was their second pregnancy. The antenatal visits of the cases of SMO grouped by resource-poor and resource-rich were 4-6 times (34.75\%) and $7-9$ times $(40.88 \%)$ differently $\left(\chi^{2}=121.42, \quad P=\right.$ $0.104)$. More SMO cases in resource-rich institutions had a history of cesarean section delivery. There was no difference in the marital status in SMO cases by hospital resource level $\left(\chi^{2}=2.65, P=0.104\right)$ (Table 2$)$.

3.3. Termination of Pregnancy among Women with SMO. Most deliveries among women with SMO were at 37-42 gestational weeks in resource-poor (56.56\%) and resource-rich (48.19\%) hospitals. In total, 111 SMO cases $(21.43 \%)$ in resource-poor hospitals and $36.26 \%$ SMO cases in resource-rich hospitals delivered low-birth-weight infants. Additionally, cesarean section was chosen in $61.39 \%$ SMO cases in resource-poor hospitals and $70.67 \%$ cases in resource-rich hospitals. Most SMO cases in resource-rich hospitals $(94.30 \%)$ had delivered at provincial hospitals, and $87.26 \%$ SMO cases in resource-poor hospitals had delivered at county hospitals. The rate of LBs in resource-rich hospitals $(92.38 \%)$ was higher than that in resource-poor hospitals (82.43\%) (Table 3).

3.4. Organ Dysfunction and Maternal Complications. Coagulation dysfunction was reported in 1021 MNM cases (78.60\%) in resource-rich hospitals and 297 MNM cases $(57.34 \%)$ in resource-poor hospitals. The common organ dysfunction associated with MD was neurologic dysfunction $(41.38 \%)$ in resource-poor hospitals and coagulation dysfunction $(42.55 \%)$ in resource-rich hospitals. The highest MI was for neurologic dysfunction in resource-poor hospitals (2.32) and coagulation dysfunction in resourcerich hospitals (1.54). The most common maternal complications in resource-poor hospitals were hypertensive disorder in $18 \mathrm{MD}$ cases and obstetric hemorrhage in 352 MNM cases. In resource-rich hospitals, the most common maternal complication was anemia (except unclassified diseases), which was found in $14 \mathrm{MD}$ cases and 877 MNM cases. The highest MI associated with maternal complications was for hypertensive disorder (3.10) in 
TABLE 1: Summary of deliveries, severe maternal outcomes, and near miss indicators in women admitted to resource-poor and resource-rich hospitals in Hunan Province from 2012 to 2018.

\begin{tabular}{|c|c|c|c|c|c|c|c|c|}
\hline Resource-poor hospitals & Total & 2012 & 2013 & 2014 & 2015 & 2016 & 2017 & 2018 \\
\hline All hospitalized pregnant women (number) & 296985 & 37227 & 40216 & 43523 & 43536 & 47883 & 47529 & 37071 \\
\hline Live births (number) & 279407 & 36095 & 38085 & 40600 & 41237 & 45250 & 44566 & 33574 \\
\hline Pregnancy complications (number) & 124791 & 4638 & 12007 & 18285 & 20061 & 23705 & 23317 & 22778 \\
\hline Severe maternal outcome cases (number) & 518 & 75 & 86 & 68 & 76 & 54 & 82 & 77 \\
\hline Maternal near miss cases (number) & 489 & 71 & 83 & 65 & 72 & 50 & 76 & 72 \\
\hline Maternal death cases (number) & 29 & 4 & 3 & 3 & 4 & 4 & 6 & 5 \\
\hline \multicolumn{9}{|l|}{ Overall near miss indicators } \\
\hline Severe maternal outcome ratio (per 1000 live births) 95\% CI & $1.85(1.70-2.02)$ & 2.08 & 2.26 & 1.67 & 1.84 & 1.19 & 1.84 & 2.29 \\
\hline Maternal near miss ratio (per 1000 live births) 95\% CI & $1.75(1.60-1.91)$ & 1.97 & 2.18 & 1.60 & 1.75 & 1.10 & 1.71 & 2.14 \\
\hline Maternal mortality ratio (per 100000 live births) 95\% CI & $10.38(6.95-14.90)$ & 11.08 & 7.88 & 7.39 & 9.70 & 8.84 & 13.46 & 14.89 \\
\hline Maternal near miss mortality ratio (MNM : MD) & 16.86 & 17.75 & 27.67 & 21.67 & 18.00 & 12.50 & 12.67 & 14.40 \\
\hline Mortality index (\%) & 5.60 & 5.33 & 3.49 & 4.41 & 5.26 & 7.41 & 7.32 & 6.49 \\
\hline Resource-rich hospitals & Total & 2012 & 2013 & 2014 & 2015 & 2016 & 2017 & 2018 \\
\hline All hospitalized pregnant women (number) & 244143 & 24659 & 26846 & 31675 & 35285 & 44175 & 43699 & 37804 \\
\hline Live births (number) & 232386 & 24086 & 25604 & 30262 & 33684 & 42108 & 41383 & 35259 \\
\hline Pregnancy complications (number) & 149997 & 4711 & 11426 & 19518 & 21912 & 29997 & 33091 & 29342 \\
\hline Severe maternal outcome cases (number) & 1299 & 111 & 175 & 182 & 168 & 222 & 200 & 241 \\
\hline Maternal near miss cases (number) & 1262 & 108 & 170 & 177 & 161 & 216 & 193 & 237 \\
\hline Maternal death cases (number) & 37 & 3 & 5 & 5 & 7 & 6 & 7 & 4 \\
\hline \multicolumn{9}{|l|}{ Overall near miss indicators } \\
\hline Severe maternal outcome ratio (per 1000 live births) 95\% CI & $5.59(5.29-5.90)$ & 4.61 & 6.83 & 6.01 & 4.99 & 5.27 & 4.83 & 6.84 \\
\hline Maternal near miss ratio (per 1000 live births) 95\% CI & $5.43(5.14-5.74)$ & 4.48 & 6.64 & 5.85 & 4.78 & 5.13 & 4.66 & 6.72 \\
\hline Maternal mortality ratio (per 100000 live births) 95\% CI & $15.92(11.51-21.95)$ & 12.46 & 19.53 & 16.52 & 20.78 & 14.25 & 16.92 & 11.34 \\
\hline Maternal near miss mortality ratio (MNM : MD) & 34.11 & 36.00 & 34.00 & 35.40 & 23.00 & 36.00 & 27.57 & 59.25 \\
\hline Mortality index (\%) & 2.85 & 2.70 & 2.86 & 2.75 & 4.17 & 2.70 & 3.50 & 1.66 \\
\hline
\end{tabular}

resource-poor hospitals and anemia (1.08) in resource-rich hospitals, excluding unclassified complications. These outcomes are shown in Table 4 and Figure 2.

\section{Discussion}

4.1. Severe Maternal Outcomes. The WHO has initiated a process of agreeing on a definition and developing a uniform set of identification criteria for MNM cases, with the intent of facilitating reviews of these cases to monitor and improve the quality of obstetric care [10]. Our study showed the prevalence of MNM was $0.175 \%$ among 296985 women attending 12 resource-poor health facilities and $0.543 \%$ among 244143 women attending five resource-rich health facilities in Hunan Province from 2012 to 2018. Other studies have reported MNM incidence rates from $0.4 \%$ to $1.6 \%$ [16-21]. A systematic review conducted in sub-Saharan Africa on the applicability of the WHO maternal near miss tool showed the median MNM ratio was 24.2 (95\% CI: 12.4-35.8) per $1000 \mathrm{LBs}$, which ranged from 4.4 in a population-based study in South Africa to 198 in a rural private hospital in Nigeria [22]. The MNM incidence among hospitals in Ethiopia was $20.8 \%$ (9.1-38.8\%), and a significant regional variation was observed with regard to MNM incidence and mortal- ity index [23]. Overall, resource-rich hospitals in China's Hunan Province treat more MNM cases, but the incidence of MNM in this study was lower than that reported in most international studies.

MD constitutes the most obvious manifestation of severe morbidity related to pregnancy, childbirth, and puerperium. Our study showed the prevalence of MD was $10.38 / 100000$ LBs (29 deaths) in the 12 resource-poor facilities and $15.92 / 100000$ LBs (37 deaths) in the five resource-rich facilities. High maternal mortality was reported in the US, with rates from 16.0 to 17.8 per 100000 LBs between 2009 and 2016 [24]. In Turkey, the MMR was 15.4 deaths per 100000 LBs in 2012, compared with 13.7 in 2015 [25]. In subSaharan Africa, the MMR was 351 per 100000 LBs, which was similar to that in South Asia (336 per 100000 LBs) [26]. Over the past few decades, substantial progress has been made in safe motherhood, possibly attributable to delivery in hospital policies and prevention and treatment of postpartum hemorrhage programs, although regional inequality persists.

Indicators such as the MNM:MD and $\mathrm{MI}$ are also used to describe obstetric care. The MNM:MD represents the proportion of MNM cases that progressed to MD, with a higher ratio reflecting better quality of care [5]. In this study, the MNM:MD was 16.86 and 34.11 in resource- 

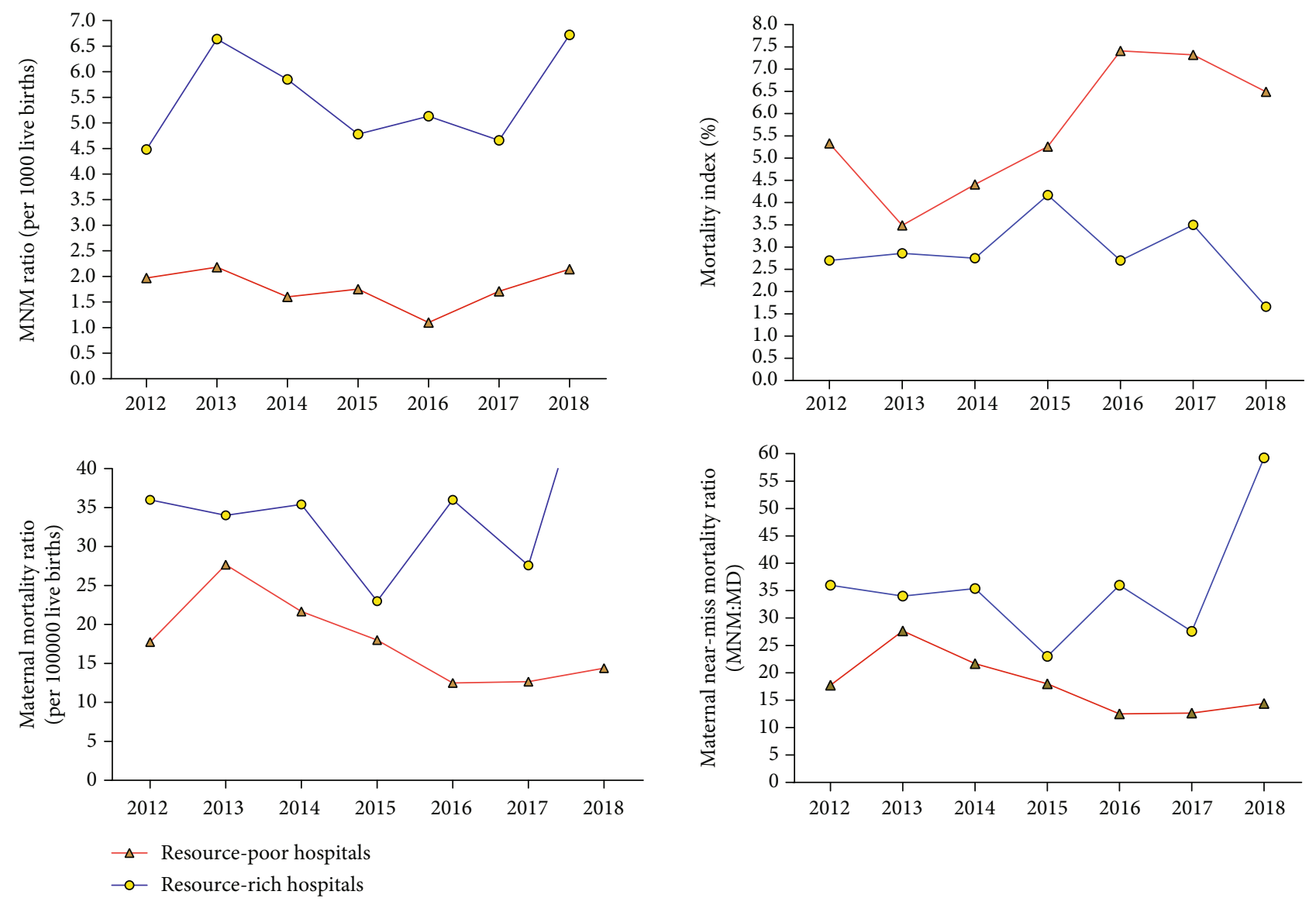

FIgURE 1: The time trend of MNM ratio, MI, maternal mortality, and MNM : MD in women admitted to resource-poor and resource-rich hospitals in Hunan Province from 2012 to 2018.

poor and resource-rich hospitals, respectively. Therefore, one MD occurred for every 17 MNM cases in resourcepoor hospitals and for every $34 \mathrm{MNM}$ cases in resourcerich hospitals. MNM cases are more likely to die in the nondeveloped and developing countries than in the developed countries. A study conducted in Somaliland and Tanzania using similar WHO criteria reported the MNM:MD was $6.7[27,28]$. However, studies conducted in South Africa such as Nigeria, Tanzania, and Ghana and Pakistan reported near ratios ranging from 1.5 to 5 $[21,26,29]$. The ratios in our study were relatively high in the context of existing policies to promote maternal and child health.

Our findings revealed a MI of 5.6\% in resource-poor hospitals and $2.85 \%$ in resource-rich hospitals, which was comparable to the $4.2 \%$ MI reported in previous studies conducted in China and Malaysia [19, 30]. A low MI $(<5 \%)$ indicates better quality of care, with fewer women with severe conditions dying; in contrast, a high MI $(>20 \%)$ indicates low-quality obstetric care for severe cases, in which more women with severe conditions die [5].

Based on our comparison of these four indicators, we concluded that resource-rich hospitals have a stronger medical treatment ability and better healthcare quality. We suggest upgrading the obstetric emergency treatment capacity in resource-poor hospitals or to redistinguish the social functions of different medical institutions, such as implementing graded diagnosis where resource-rich hospitals focus on treating MNM cases, and resource-poor hospitals focus on monitoring basic health indictors for pregnant women in rural areas and city communities based on the current medical resource and level of critical illness treatment. Results from our analysis may contribute to the development of the healthcare system in China.

4.2. Demographic Differences and Birth Outcomes. This study mainly compared women admitted to resource-poor hospitals with those admitted to resource-rich hospitals. Overall, the proportion of giving birth to a second child was highest in SMO cases. The phenomenon is due to the background of two-child policy opening and high cesarean section rate in China. The main differences found in the demographic comparisons were in the number of antenatal visits and history of cesarean section. SMO cases in resource-rich hospitals had more antenatal visits and a higher proportion of cesarean sections than SMO cases in resource-poor hospitals. In addition, $33.35 \%$ of all SMO cases were women with a history of cesarean section, which was less than the $58 \%$ reported in a study at a tertiary referral teaching hospital in the southeast of Iran 
TABLE 2: Personal information for women with severe maternal outcomes at resource-poor and resource-rich hospitals in Hunan Province from 2012 to 2018.

\begin{tabular}{|c|c|c|c|c|c|c|}
\hline \multirow[t]{2}{*}{ Variables } & \multicolumn{2}{|c|}{$\begin{array}{l}\text { Resource- } \\
\text { poor } \\
\text { hospitals }\end{array}$} & \multicolumn{2}{|c|}{$\begin{array}{l}\text { Resource- } \\
\text { rich } \\
\text { hospitals }\end{array}$} & \multirow[t]{2}{*}{$\chi^{2}$} & \multirow[t]{2}{*}{$P$} \\
\hline & $N$ & $\%$ & $N$ & $\%$ & & \\
\hline \multicolumn{7}{|l|}{ Mother's age (years) } \\
\hline$<20$ & 17 & 3.28 & 13 & 1.00 & \multirow{7}{*}{35.263} & \multirow{7}{*}{$<0.001$} \\
\hline $20-24$ & 68 & 13.13 & 100 & 7.70 & & \\
\hline $25-29$ & 149 & 28.76 & 405 & 31.18 & & \\
\hline $30-34$ & 126 & 24.32 & 440 & 33.87 & & \\
\hline $35-39$ & 80 & 15.44 & 222 & 17.09 & & \\
\hline$\geq 40$ & 33 & 6.37 & 91 & 7.01 & & \\
\hline Missing & 45 & 8.69 & 28 & 2.16 & & \\
\hline \multicolumn{7}{|l|}{ Mother's education } \\
\hline None/primary school & 30 & 5.79 & 21 & 1.62 & \multirow{5}{*}{318.1} & \multirow{5}{*}{$<0.001$} \\
\hline Middle school & 187 & 36.10 & 117 & 9.01 & & \\
\hline High school & 249 & 48.07 & 637 & 49.04 & & \\
\hline College or higher & 34 & 6.56 & 504 & 38.80 & & \\
\hline Missing & 18 & 3.47 & 20 & 1.54 & & \\
\hline \multicolumn{7}{|l|}{ Marital status } \\
\hline $\begin{array}{l}\text { Single, widowed, } \\
\text { or divorced }\end{array}$ & 16 & 3.09 & 24 & 1.85 & \multirow[t]{2}{*}{2.65} & \multirow[t]{2}{*}{0.104} \\
\hline Married & 502 & 96.91 & 1275 & 98.15 & & \\
\hline \multicolumn{7}{|l|}{ Parity } \\
\hline 0 & 181 & 34.94 & 544 & 41.88 & \multirow{3}{*}{9.063} & \multirow{3}{*}{0.011} \\
\hline 1 & 265 & 51.16 & 618 & 47.58 & & \\
\hline$\geq 2$ & 72 & 13.90 & 137 & 10.55 & & \\
\hline \multicolumn{7}{|l|}{ Antenatal visits } \\
\hline 0 & 15 & 2.90 & 14 & 1.08 & \multirow{6}{*}{121.42} & \multirow{6}{*}{$<0.001$} \\
\hline $1-3$ & 87 & 16.80 & 116 & 8.93 & & \\
\hline $4-6$ & 180 & 34.75 & 294 & 22.63 & & \\
\hline $7-9$ & 125 & 24.13 & 531 & 40.88 & & \\
\hline$\geq 10$ & 42 & 8.11 & 296 & 22.79 & & \\
\hline Missing & 68 & 13.13 & 48 & 3.70 & & \\
\hline \multicolumn{7}{|c|}{ History of cesarean sections } \\
\hline Yes & 138 & 26.64 & 468 & 36.03 & \multirow{2}{*}{14.679} & \multirow{2}{*}{$<0.001$} \\
\hline No & 380 & 73.36 & 831 & 63.97 & & \\
\hline
\end{tabular}

[31]. Although previous cesarean delivery is not considered a risk factor for SMO or adverse perinatal outcomes, there could be differences between study groups in terms of healthcare-seeking behavior, referral mechanisms, intrapartum monitoring, and clinical decision-making [32]. Considering that resource-poor hospitals are located in rural areas and resource-rich hospitals are located in urban areas, there were fewer antenatal visits for pregnant women living in rural areas. This may be related to health awareness. It may be necessary to improve maternal health knowledge and focus on behavior change in low-resource and limited literacy settings, which may contribute to reductions in maternal mortality [33].
TABLE 3: Termination of pregnancy for cases with severe maternal outcomes at resource-poor and resource-rich hospitals in Hunan Province from 2012 to 2018.

\begin{tabular}{|c|c|c|c|c|}
\hline \multirow[t]{2}{*}{ Variables } & \multicolumn{2}{|c|}{$\begin{array}{c}\text { Resource- } \\
\text { poor hospitals }\end{array}$} & \multicolumn{2}{|c|}{$\begin{array}{c}\text { Resource-rich } \\
\text { hospitals }\end{array}$} \\
\hline & $N$ & $\%$ & $N$ & $\%$ \\
\hline \multicolumn{5}{|l|}{ Gestational age (weeks) } \\
\hline$<28$ & 52 & 10.04 & 107 & 8.24 \\
\hline $29-36$ & 105 & 20.27 & 505 & 38.88 \\
\hline $37-42$ & 293 & 56.56 & 626 & 48.19 \\
\hline Missing & 68 & 13.13 & 61 & 4.70 \\
\hline \multicolumn{5}{|l|}{ Birth weight (grams) } \\
\hline$<2500$ & 111 & 21.43 & 471 & 36.26 \\
\hline $2500-4000$ & 298 & 57.53 & 744 & 57.27 \\
\hline$\geq 4000$ & 32 & 6.18 & 51 & 3.93 \\
\hline Missing & 103 & 19.88 & 145 & 11.16 \\
\hline \multicolumn{5}{|c|}{ Mode of delivery/end of pregnancy } \\
\hline Vaginal delivery & 124 & 23.94 & 284 & 21.86 \\
\hline Cesarean section & 318 & 61.39 & 918 & 70.67 \\
\hline Abortion/others & 50 & 9.65 & 62 & 4.77 \\
\hline Missing & 26 & 5.02 & 35 & 2.69 \\
\hline \multicolumn{5}{|l|}{ Place of delivery } \\
\hline Provincial hospital & 4 & 0.77 & 1225 & 94.30 \\
\hline County hospital & 452 & 87.26 & 19 & 1.46 \\
\hline Township hospital/others & 25 & 4.83 & 19 & 1.46 \\
\hline Missing & 37 & 7.14 & 36 & 2.77 \\
\hline \multicolumn{5}{|l|}{ Total deliveries } \\
\hline 1 & 459 & 88.61 & 1136 & 87.45 \\
\hline$\geq 2$ & 26 & 5.02 & 111 & 8.55 \\
\hline Unknown/missing & 33 & 6.37 & 52 & 4.00 \\
\hline \multicolumn{5}{|l|}{ Gender } \\
\hline Female & 244 & 47.10 & 645 & 49.65 \\
\hline Male & 225 & 43.44 & 642 & 49.42 \\
\hline Unknown/missing & 75 & 14.48 & 125 & 9.62 \\
\hline \multicolumn{5}{|l|}{ State of birth } \\
\hline Live birth & 427 & 82.43 & 1200 & 92.38 \\
\hline Stillbirth & 44 & 8.49 & 112 & 8.62 \\
\hline Unknown/missing & 73 & 14.09 & 100 & 7.70 \\
\hline
\end{tabular}

Among the 1817 SMO cases in this study, $51 \%$ cases delivered at 37-42 gestational weeks, $68 \%$ cases delivered by cesarean section, and $88 \%$ of women were giving birth for the first time. Most newborns were LBs and term infants. Several studies have shown that the presence of MNM in women is strongly associated with the occurrence of adverse perinatal outcomes, such as stillbirth, preterm birth, low birth weight, and early neonatal mortality [34-37]. However, studies that quantified the effect of MNM on adverse perinatal outcomes are scarce in China. We intend to explore this issue in a further study.

4.3. Clinical Disorders. Hypertensive disorders and neurologic dysfunction were responsible for most MD cases in resource-poor hospitals, whereas anemia and coagulation 


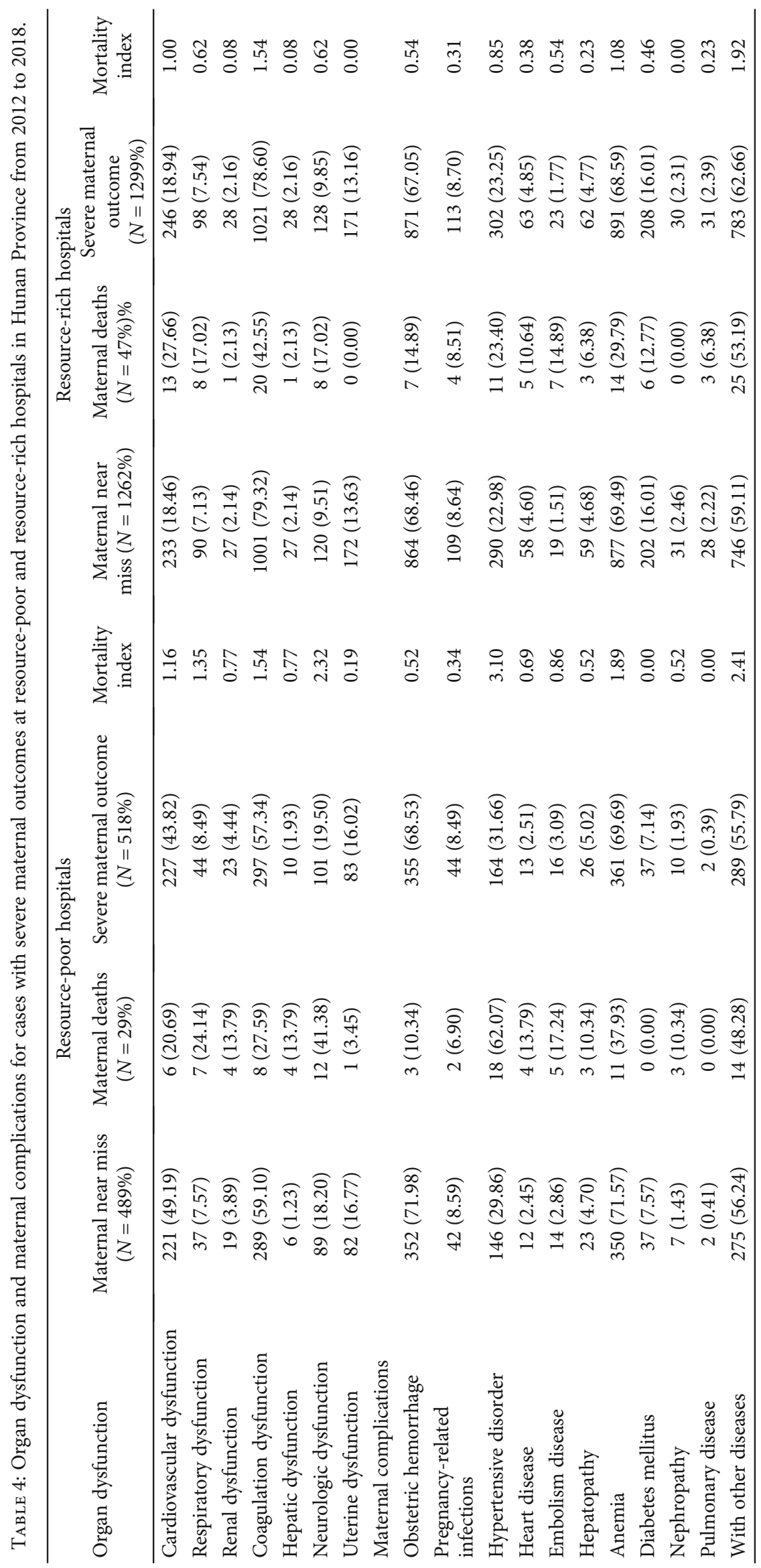




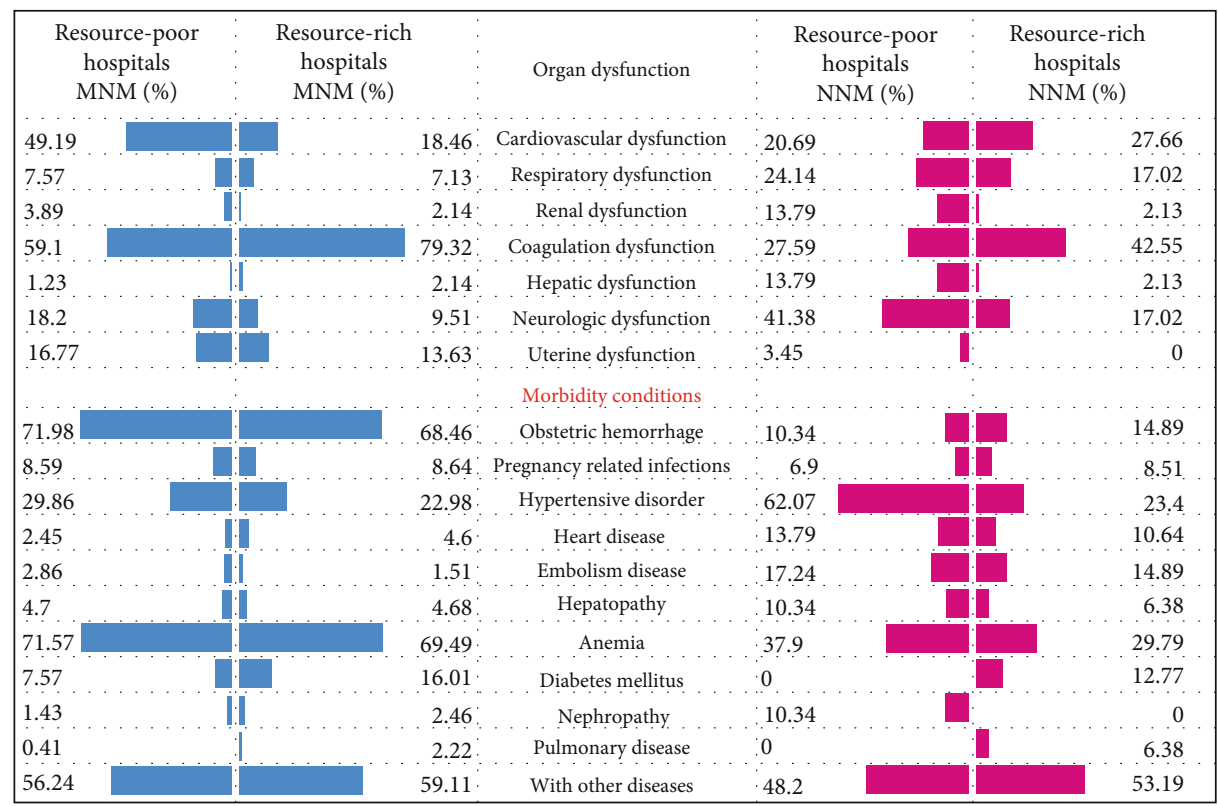

Figure 2: The proportion of organ dysfunction and maternal complications for MNM and MD cases at resource-poor and resource-rich hospitals in Hunan Province from 2012 to 2018.

dysfunction were most common in MD cases in resourcerich hospitals. However, for MNM cases, anemia and coagulation dysfunction were the leading cause of death in both resource-poor and resource-rich hospitals. We found the major causes of SMO were anemia, hypertensive disorder, and obstetric hemorrhage. Hypertensive disorder and cardiovascular dysfunction were the most frequent conditions among MNM cases in Alborz Province of Iran, where medical resources are undeveloped [38]. In the setting of the present study, hypertensive disorders related to MNM cases in resource-poor hospitals and anemia in resource-rich hospitals need more attention to prevent SMO cases in Hunan Province. Anemia in pregnancy is associated with severe maternal morbidity and is an indirect cause of $\mathrm{MD}$ across pregnant populations in both low- and high-income settings [39]. The high prevalence of anemia also contributed to adverse outcomes in women with prenatal hemorrhage. It is therefore crucial to optimize maternal iron status to improve maternal outcomes [40]. Postpartum hemorrhage was less common in the present study compared with other studies, which may be because of the high coverage of essential medical interventions to prevent this condition.

\section{Limitations}

We applied the unified WHO maternal near miss criteria in different-sized hospitals, which might have resulted in information bias because of differences in diagnostic capabilities and standards. Second, we used facility-based sampling, which could have introduced referral bias. Third, some related risk factors associated with MNM such as prepregnancy body mass index, diseases before pregnancy, and family history were not collected in this study.

\section{Conclusions}

The study shows higher ratios of MNM and MD cases in resource-rich hospitals compared with resource-poor hospitals. The obstetric emergency capacity of resource-rich hospitals is higher than that of resource-poor hospitals. Our findings suggest future government policies should consider upgrading the obstetric emergency treatment capacity in resource-poor hospitals or to redistinguish the social functions of different medical institutions.
Abbreviations
SMO: $\quad$ Severe maternal outcomes
MNM: $\quad$ Maternal near miss
MD: $\quad$ Maternal deaths
MI: $\quad$ Mortality index
MNM : MD: The ratio of maternal near miss to mortality
LBs: $\quad$ Live births
NMNMSS: China's National Maternal Near Miss Surveil-
WHO: $\quad$ World Health Organization
CI: $\quad$ Confidence intervals
MMR: $\quad$ Maternal mortality ratio.

\section{Data Availability}

The datasets used to support the findings of this study are included within the article.

\section{Conflicts of Interest}

The authors declare that they have no competing interests. 


\section{Authors' Contributions}

XLL was primarily responsible for conceiving the project and writing the paper. ZMJ was responsible for modifying the paper. $\mathrm{WAH}, \mathrm{XDH}$, and $\mathrm{KFJ}$ were responsible for carrying out data collection and analysis of the manuscript. LZY was responsible for reviewing the manuscript. Xiong Lili and Zeng Mengjun were the coauthors.

\section{Acknowledgments}

We thank Audrey Holmes, MA, from Liwen Bianji, Edanz Group China (http://www.liwenbianji.cn/ac), for editing the English text of a draft of this manuscript. We wish to thank the staffs who had collected, recorded, and reviewed the information of maternal near miss surveillance relevant to this study. The manuscript has been submitted to Research Square.

\section{References}

[1] Global, regional, and national levels of maternal mortality, 1990-2015: a systematic analysis for the Global Burden of Disease Study 2015, 2016.

[2] Y. Gao, H. Zhou, N. S. Singh et al., "Progress and challenges in maternal health in western China: a countdown to 2015 national case study," The Lancet Global Health, vol. 5, no. 5, pp. e523-e536, 2017.

[3] X. Lili, H. Jian, Z. Mengjun et al., "Epidemiological analysis of maternal deaths in Hunan Province in China between 2009 and 2014," PLoS One, vol. 13, no. 11, p. e0207920, 2018.

[4] M. C. Hogan, K. J. Foreman, M. Naghavi et al., "Maternal mortality for 181 countries, 1980-2008: a systematic analysis of progress towards Millennium Development Goal 5," The Lancet, vol. 375, no. 9726, pp. 1609-1623, 2010.

[5] Organization WH, Evaluating the quality of care for severe pregnancy complications: the WHO near-miss approach for maternal health, 2011.

[6] V. Filippi, D. Chou, M. Barreix, and L. Say, "A new conceptual framework for maternal morbidity," International Journal of Gynecology \& Obstetrics, vol. 141, no. 3, pp. 4-9, 2018.

[7] A. Bacci, G. Lewis, V. Baltag, and A. P. Betrán, "The introduction of confidential enquiries into maternal deaths and nearmiss case reviews in the WHO European Region," Reproductive Health Matters, vol. 15, no. 30, pp. 145-152, 2007.

[8] R. C. Pattinson and M. Hall, "Near misses: a useful adjunct to maternal death enquiries," British Medical Bulletin, vol. 67, no. 1, pp. 231-243, 2003.

[9] S. E. Geller, D. Rosenberg, S. M. Cox et al., "The continuum of maternal morbidity and mortality: factors associated with severity," American Journal of Obstetrics and Gynecology, vol. 191, no. 3, pp. 939-944, 2004.

[10] L. Say, J. P. Souza, and R. C. Pattinson, "Maternal near misstowards a standard tool for monitoring quality of maternal health care," Best Practice \& Research Clinical Obstetrics \& Gynaecology, vol. 23, no. 3, pp. 287-296, 2009.

[11] J. P. Souza, A. M. Gülmezoglu, J. Vogel et al., "Moving beyond essential interventions for reduction of maternal mortality (the WHO Multicountry Survey on Maternal and Newborn Health): a cross-sectional study," The Lancet, vol. 381, no. 9879, pp. 1747-1755, 2013.
[12] L. Say, R. C. Pattinson, and A. M. Gülmezoglu, "WHO systematic review of maternal morbidity and mortality: the prevalence of severe acute maternal morbidity (near miss)," Reproductive Health, vol. 1, no. 1, p. 3, 2004.

[13] Ö. Tunçalp and J. Souza, "Maternal near-miss audits to improve quality of care," Bjog An International Journal of Obstetrics \& Gynaecology, vol. 121, Suppl 4, pp. 102-104, 2014.

[14] L. Alkema, D. Chou, D. Hogan et al., "Global, regional, and national levels and trends in maternal mortality between 1990 and 2015, with scenario-based projections to 2030: a systematic analysis by the UN Maternal Mortality Estimation Inter-Agency Group," The Lancet, vol. 387, no. 10017, pp. 462-474, 2016.

[15] J. Zhu, J. Liang, Y. Mu et al., "Sociodemographic and obstetric characteristics of stillbirths in China: a census of nearly 4 million health facility births between 2012 and 2014," The Lancet Global Health, vol. 4, no. 2, pp. e109-e118, 2016.

[16] A. J. Pacheco, L. Katz, A. S. Souza, and M. M. de Amorim, "Factors associated with severe maternal morbidity and near miss in the São Francisco Valley, Brazil: a retrospective, cohort study," BMC Pregnancy and Childbirth, vol. 14, no. 1, 2014.

[17] L. P. L. Galvão, F. Alvim-Pereira, C. M. M. D. Mendonça, F. E. F. Menezes, and R. Q. Gurgel, "The prevalence of severe maternal morbidity and near miss and associated factors in Sergipe, Northeast Brazil," BMC Pregnancy \& Childbirth, vol. 14, no. 1, p. 25, 2014.

[18] A. P. Madeiro, A. C. Rufino, É. Z. G. Lacerda, and L. G. Brasil, "Incidence and determinants of severe maternal morbidity: a transversal study in a referral hospital in Teresina, Piaui, Brazil," BMC Pregnancy and Childbirth, vol. 15, no. 1, 2015.

[19] M. N. Norhayati, N. H. Nik Hazlina, Z. Sulaiman, and M. Y. Azman, "Severe maternal morbidity and near misses in tertiary hospitals, Kelantan, Malaysia: a cross-sectional study," BMC Public Health, vol. 16, p. 229, 2016.

[20] J. P. Souza, A. M. Gülmezoglu, J. Vogel et al., "Moving beyond essential interventions for reduction of maternal mortality (the WHO Multicountry Survey on Maternal and Newborn Health): a cross-sectional study," Lancet, vol. 381, no. 9879, pp. 1747-1755, 2013.

[21] O. Oladapo, O. Adetoro, B. Ekele et al., "When getting there is not enough: a nationwide cross-sectional study of 998 maternal deaths and 1451 near-misses in public tertiary hospitals in a low-income country," BJOG: An International Journal of Obstetrics \& Gynaecology, vol. 123, no. 6, pp. 928-938, 2016.

[22] A. K. Tura, T. L. Trang, T. van den Akker et al., “Applicability of the WHO maternal near miss tool in sub-Saharan Africa: a systematic review," BMC Pregnancy and Childbirth, vol. 19, no. 1, p. 79, 2019.

[23] A. Geleto, C. Chojenta, T. Taddele, and D. Loxton, "Incidence of maternal near miss among women in labour admitted to hospitals in Ethiopia,” Midwifery, vol. 82, p. 102597, 2020.

[24] E. E. Petersen, N. L Davis, D. Goodman et al., "Racial/Ethnic Disparities in Pregnancy-Related Deaths - United States, 2007-2016," MMWR. Morbidity and Mortality Weekly Report, vol. 68, no. 35, pp. 762-765, 2019.

[25] Y. Engin-Üstün, S. Sanisoğlu, H. L. Keskin et al., "Changing trends in the Turkish maternal deaths, with a focus on direct and indirect causes," European Journal of Obstetrics \& Gynecology and Reproductive Biology, vol. 234, pp. 21-25, 2019. 
[26] I. Ahmed, S. M. Ali, S. Amenga-Etego et al., "Population-based rates, timing, and causes of maternal deaths, stillbirths, and neonatal deaths in South Asia and sub-Saharan Africa: a multi-country prospective cohort study," The Lancet Global Health, vol. 6, no. 12, pp. e1297-e1308, 2018.

[27] J. Kiruja, F. Osman, J. A. Egal, B. Essén, M. Klingberg-Allvin, and K. Erlandsson, "Maternal near-miss and death incidences-frequencies, causes and the referral chain in Somaliland: a pilot study using the WHO near-miss approach," Sexual \& Reproductive Healthcare, vol. 12, pp. 30-36, 2017.

[28] E. Nelissen, E. Mduma, J. Broerse et al., "Applicability of the WHO maternal near miss criteria in a low-resource setting," PLoS One, vol. 8, no. 4, article e61248, 2013.

[29] Ö. Tunçalp, M. J. Hindin, K. Adu-Bonsaffoh, and R. M. Adanu, "Understanding the continuum of maternal morbidity in Accra, Ghana," Maternal \& Child Health Journal, vol. 18, no. 7, pp. 1648-1657, 2014.

[30] F. R. Shen, M. Liu, X. Zhang, W. Yang, and Y. G. Chen, "Factors associated with maternal near-miss morbidity and mortality in Kowloon Hospital, Suzhou, China," International Journal of Gynaecology and Obstetrics: The Official Organ of the International Federation of Gynaecology and Obstetrics, vol. 123, no. 1, pp. 64-67, 2013.

[31] Z. Moudi, L. Arabnezhad, H. Ansari, and S. M. Tabatabaei, "Severe maternal morbidity among women with a history of cesarean section at a tertiary referral teaching hospital in the southeast of Iran," Public Health, vol. 175, pp. 101-107, 2019.

[32] H. Litorp, M. Rööst, H. L. Kidanto, L. Nyström, and B. Essén, "The effects of previous cesarean deliveries on severe maternal and adverse perinatal outcomes at a university hospital in Tanzania," International Journal of Gynecology \& Obstetrics, vol. 133, no. 2, pp. 183-187, 2016.

[33] B. B. Sharma, D. J. Loxton, H. Murray et al., "A first step to improving maternal mortality in a low-literacy setting; the successful use of singing to improve knowledge regarding antenatal care," American Journal of Obstetrics and Gynecology, vol. 219, no. 6, p. 615.e611, 2018.

[34] L. C. Oliveira and A. A. Ribeiro da Costa, "Fetal and neonatal deaths among cases of maternal near miss," Revista da Associação Médica Brasileira (English Edition), vol. 59, no. 5, pp. 487494, 2013.

[35] A. C. Ramirez, F. Kouyoumdjian, J. Liauw, D. Costescu, H. Lu, and L. Holder, "Infant and maternal outcomes in women who experience imprisonment in Ontario, Canada: a retrospective cohort study," Journal of Obstetrics and Gynaecology Canada, vol. 41 , no. 5 , p. 714, 2019.

[36] O. O. Owolabi, J. A. Cresswell, B. Vwalika, D. Osrin, and V. Filippi, "Incidence of abortion-related near-miss complications in Zambia: cross-sectional study in Central, Copperbelt and Lusaka Provinces," Contraception, vol. 95, no. 2, pp. 167-174, 2017.

[37] E. F. Liyew, A. W. Yalew, M. F. Afework, and B. Essen, "Maternal near-miss and the risk of adverse perinatal outcomes: a prospective cohort study in selected public hospitals of Addis Ababa, Ethiopia," BMC Pregnancy and Childbirth, vol. 18, no. 1, p. 345, 2018.

[38] Z. Ghazivakili, R. Lotfi, and K. Kabir, "Maternal near miss approach to evaluate quality of care in Alborz Province, Iran," Midwifery, vol. 41, pp. 118-124, 2016.
[39] J. Daru, J. Zamora, B. M. Fernández-Félix et al., "Risk of maternal mortality in women with severe anaemia during pregnancy and post partum: a multilevel analysis," The Lancet Global Health, vol. 6, no. 5, pp. e548-e554, 2018.

[40] R. Mustafa and H. Hashmi, "Near-miss obstetrical events and maternal deaths," Journal of College of Physicians And Surgeons Pakistan, vol. 19, no. 12, pp. 781-785, 2009. 